\title{
Congrès général SFP à Grenoble: haut niveau et ouverture
}

On entend dire que les congrès généraux des sociétés savantes perdent de leur intérêt : les jeunes préfèrent les rencontres de leur spécialité pour assurer leur carrière, les seniors manquent de temps avec la multiplication des invitations à l'étranger; et, de toute façon, il y a tant d'autres canaux pour s'informer sur les progrès de la science... Avant chaque congrès général de la SFP se pose donc la question de la pertinence de cette manifestation traditionnelle. Pourtant cette année, de l'avis général, les quelques jours du congrès en juillet à Grenoble ont été un

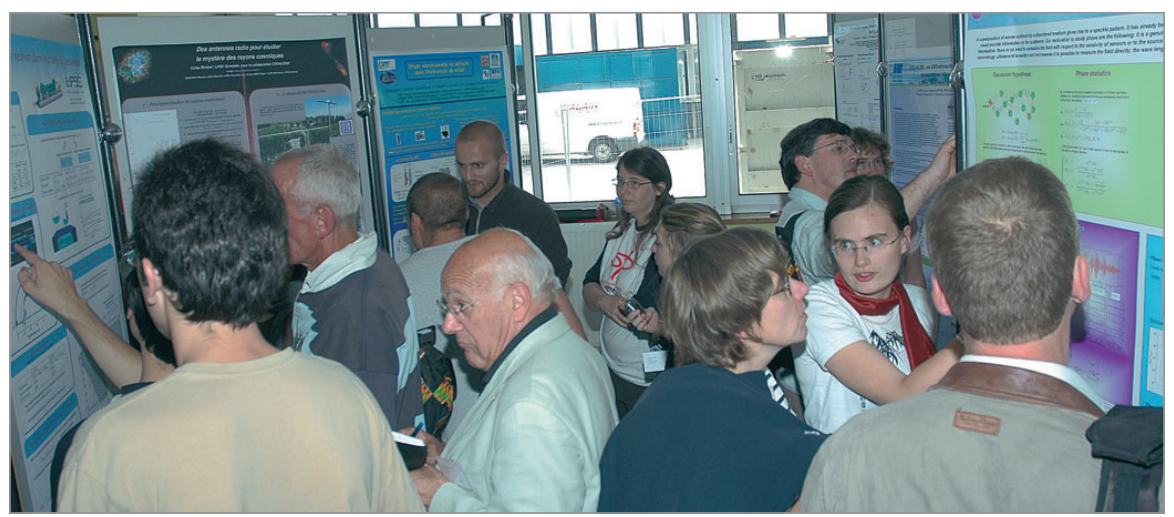

On a pu à l'occasion mesurer que la qualité d'un exposé en séance plénière n'est pas réservée aux chercheurs chevronnés, puisque c'est un tout jeune astrophysicien qui nous a communiqué son enthousiasme pour les exo-planètes. Et je n'ai garde d'oublier le vif succès grand public d'André Brahic au Musée des Beaux-Arts, avec sa conférence d'astrophysique et ses superbes images de Titan.

Les tables rondes ont constitué d'autres points forts du congrès de Grenoble. L'emploi des jeunes docteurs reste toujours une vive préoccupation. Le thème de la place de la physique dans les écoles d'ingénieurs, abordé pour la première fois, sera étudié plus en détail par la SFP sous la responsabilité de Vincent Mosser. C'est toutefois le débat sur l'énergie et l'environnement qui a attiré authentique succès. L'audience est restée présente tout au long de la semaine, les jeunes sont venus nombreux et s'y sont montrés très participatifs. Avant tout, je voudrais ici remercier le président du congrès Alain Aspect et l'équipe des organisateurs grenoblois emmenée par Etienne Wolf, pour la pertinence de leurs choix qui ont donné aux sessions un haut niveau scientifique en même temps qu'une ouverture bienvenue sur l'actualité.

Ce congrès restera comme celui qui a suivi de très peu la disparition de Pierre-Gilles de Gennes. Un hommage émouvant lui a été rendu par Jacques Prost, qui a rappelé comment, avec une vision très globale de la physique, il a su rapprocher élégamment la supraconductivité et les cristaux liquides. L'histoire de la supraconductivité aura également été évoquée avec un autre témoignage marquant, celui de Philippe Nozières : ayant vécu à Princeton la naissance de la théorie BCS, il nous en a raconté les péripéties scientifiques et humaines avec beaucoup de finesse. Les conférences invitées, très généralement fort bien présentées, formaient un ensemble varié. la participation du public la plus massive, en fonction du choix judicieux des intervenants choisis par Hervé Nifenecker et des exposés plus proprement scientifiques et très pluridisciplinaires ayant précédé la table ronde.

Si la formule de ce congrès a particulièrement plu aux jeunes chercheurs cette année, c'est d'abord que les séances de posters n'étaient pas en parallèle avec d'autres sessions. Le concours pour les meilleurs posters a eu naturellement du succès. En outre une innovation expérimentée au cours de ce congrès s'est révélée très populaire : des prix ont récompensé trois exposés présentés par des doctorants, dont la maîtrise n'a pas laissé d'impressionner l'auditoire.

L'assemblée générale s'est finalement interrogée sur la date et la forme du prochain congrès général de la SFP. II devrait se tenir en septembre 2009 en Île-de-France et être parrainé par le "Triangle de la physique » du plateau de Saclay.

Michèle Leduc 